\title{
Conjugacy for positive permutation braids
}

\author{
by \\ Hugh R. Morton and Richard J. Hadji (Liverpool)
}

\begin{abstract}
Positive permutation braids on $n$ strings, which are defined to be positive $n$-braids where each pair of strings crosses at most once, form the elementary but nontrivial building blocks in many studies of conjugacy in the braid groups. We consider conjugacy among these elementary braids which close to knots, and show that those which close to the trivial knot or to the trefoil are all conjugate. All such $n$-braids with the maximum possible crossing number are also shown to be conjugate.

We note that conjugacy of these braids for $n \leq 5$ depends only on the crossing number. In contrast, we exhibit two such braids on 6 strings with 9 crossings which are not conjugate but whose closures are each isotopic to the $(2,5)$ torus knot.
\end{abstract}

Introduction. Braids have been used for many years now as a means of studying knots and links. A geometric $n$-braid joins $n$ standard reference points in $\mathbb{R}^{2}$ at the top and bottom of $\mathbb{R}^{2} \times I$ by disjointly embedded strings which are monotonic in the $I$ parameter. Concatenation of these braids leads to Artin's well established group structure on isotopy classes of geometric $n$-braids, forming the braid group $B_{n}$ (see [1]).

The closure of a braid is constructed by joining the top and bottom points in a standard way, to form a knot or link. An old result of Alexander shows that every link can be presented as the closure of some braid, while a theorem of Markov shows that any two braids presenting the same link are related by a sequence of moves of two types (see for example [2]).

One of these two moves is conjugacy of braids. Indeed it is not difficult to show that conjugate braids close to equivalent links, so the "conjugacy problem" in the braid group $B_{n}$ is of immediate interest in studying closed braids.

Algorithms for the conjugacy problem, to decide whether two given elements of $B_{n}$ are conjugate, have been developed, starting with the work of

2000 Mathematics Subject Classification: Primary 57M25.

Key words and phrases: positive permutation braids, conjugacy, cycles.

The second author was supported by EPSRC grant 99801479. 
Garside $[8,5,7]$. In many cases they depend on writing the braid in terms of a minimal number of "positive permutation braids".

In this paper we examine the position of braids which are just single positive permutation braids, and address the resulting problem of deciding when two positive permutation braids on $n$ strings are conjugate.

A necessary condition is that the corresponding permutations be conjugate, in other words the permutations have the same cycle type. In this investigation we restrict ourselves to the case where the closure of the braid is a knot, and equivalently to those permutations in $S_{n}$ which are $n$-cycles.

While any two such permutations are conjugate, the corresponding permutation braids need not be. A sufficient condition is that the closures of the braids be isotopic as closed braids, in other words the closed braids must be isotopic in the solid torus which is the complement of the braid axis [11].

We examine how far this condition follows from weaker necessary conditions on the braids, proving the following theorems:

Theorem 2. Positive permutation braids on $n$ strings which close to the unknot are all conjugate.

Theorem 3. Positive permutation braids on $n$ strings which close to the trefoil are all conjugate.

Theorem 4. Positive permutation braids on $n$ strings which close to the same knot are all conjugate, when $n \leq 5$.

We also prove a general result in Theorem 5 about conjugacy of such braids which have the largest possible number of crossings. On the other hand in Theorem 6 we exhibit two 6 -string positive permutation braids which close to the $(2,5)$ torus knot but are not conjugate. These are constructed along the lines of Murasugi and Thomas' original example of nonconjugate positive braids with isotopic closure [13].

Further simple non-conjugacy results in Theorem 7 give a range of nonconjugate positive braids closing to the trefoil, in contrast to Theorem 3 .

Some of our results were first noted in [9] by the second author. There has also been a recent exploration by Elrifai and Benkhalifa [4] for small values of $n$ without restrictions on the cycle type of the permutation.

The techniques used in this paper to prove non-conjugacy are very direct; more subtle techniques, such as Fiedler's Gauss sum invariants [6] or the algorithm of Franco and González-Meneses [7], may be used in more difficult cases. Hall has examples coming from the realms of dynamical systems of positive permutation braids on 12 or more strings which are believed not to be conjugate to their reverse [10]. In such cases none of the techniques used here can be applied to establish non-conjugacy. 
1. Permutation braids. We shall use Artin's classical description of the group $B_{n}$ of braids on $n$ strings in terms of elementary generators $\sigma_{i}$ for $i=1, \ldots, n-1$ with the relations:

1. $\sigma_{i} \sigma_{j}=\sigma_{j} \sigma_{i}$ for $|i-j| \geq 2$,

2. $\sigma_{i} \sigma_{i+1} \sigma_{i}=\sigma_{i+1} \sigma_{i} \sigma_{i+1}$ for $1 \leq i \leq n-2$.

There are two simple homomorphisms from the group $B_{n}$ which give initial constraints on conjugacy:

- The homomorphism $\varphi: B_{n} \rightarrow S_{n}$ defined on the generators by $\varphi\left(\sigma_{i}\right)=(i i+1)$ determines a permutation $\pi=\varphi(\beta)$ in which $\pi(j)$ gives the endpoint of the string of $\beta$ which begins at $j$.

- The homomorphism wr : $B_{n} \rightarrow \mathbb{Z}$ defined by $\operatorname{wr}\left(\sigma_{i}\right)=1$ counts the writhe or "algebraic crossing number" of a braid.

Two conjugate braids in $B_{n}$ must then have the same writhe, since $\mathbb{Z}$ is abelian, and also have permutations of the same cycle type.

Definition. A positive braid is an element of $B_{n}$ which can be written as a word in positive powers of the generators $\left\{\sigma_{i}\right\}$, without use of the inverse elements $\sigma_{i}^{-1}$.

For positive braids, the writhe is simply the number of crossings in the braid.

Definition. A braid $\beta$ is called a positive permutation braid if it is a positive braid such that no pair of strings cross more than once.

Notation. We denote the set of positive braids and positive permutation braids in $B_{n}$ by $B_{n}^{+}$and $S_{n}^{+}$respectively.

This definition of positive permutation braids was first used by Elrifai in $[3,5]$, where they were shown to correspond exactly to permutations. Explicitly, the homomorphism $\varphi$ restricts to a bijection from the set $S_{n}^{+}$of positive permutation braids to $S_{n}$. They were also identified by Elrifai with the set of initial segments of Garside's fundamental braid $\Delta_{n}$.

It should be noted that the explicit braid word for a positive permutation braid is generally not unique. For example, the permutation (1423) can be represented in $S_{4}^{+}$by braid words $\sigma_{1} \sigma_{2} \sigma_{3} \sigma_{1} \sigma_{2}$ and $\sigma_{2} \sigma_{1} \sigma_{3} \sigma_{2} \sigma_{3}$. Consequently, some authors choose to label permutation braids simply by the corresponding permutation in $S_{n}$.

The number of components of the closure $\widehat{\beta}$ of a braid $\beta$, constructed by identifying the initial points with the end points, is the number of cycles in the cycle type of the permutation $\varphi(\beta)$. In this paper we restrict attention to braids which close to knots, and hence we shall only look at the $(n-1)$ ! permutation braids whose permutation is a single $n$-cycle. 
2. Conjugacy results. Suppose that two braids $\beta$ and $\gamma$ are conjugate in $B_{n}$. Then their closures are isotopic as links in the complement of the braid axis, and so they are certainly isotopic in $S^{3}$.

Where $\beta$ and $\gamma$ are positive they must have the same number of crossings, because they have the same writhe. Even if they are not conjugate, two positive braids in $B_{n}$ which close to isotopic knots must have the same number of crossings.

LEMmA 1. If $\beta, \gamma \in B_{n}^{+}$and $\widehat{\beta}, \widehat{\gamma}$ are isotopic knots then $\operatorname{wr}(\beta)=\operatorname{wr}(\gamma)$.

Proof. Suppose that the knot $\widehat{\beta}$ has genus $g$. The closure of a non-split positive braid $\beta \in B_{n}$ is always a fibred link or knot. The surface found from $\widehat{\beta}$ by Seifert's algorithm is a fibre surface, and has minimal genus $g$. Its Euler characteristic $\chi=1-2 g$ satisfies $1-\chi=c-(n-1)$ where $c=\operatorname{wr}(\beta)$ is the number of crossings in $\beta$. Hence $\operatorname{wr}(\beta)=(n-1)+2 g=\operatorname{wr}(\gamma)$, since $\widehat{\gamma}$ is isotopic to $\widehat{\beta}$ and so also has genus $g$.

Consequently, if a positive $n$-braid closes to the unknot then it must have exactly $n-1$ crossings. If it closes to the trefoil knot, which has genus 1 , then it must have $n+1$ crossings. We now show that positive permutation $n$-braids which close to either of these knots are determined up to conjugacy.

Explicitly we have the following results.

TheOREm 2. Any positive permutation $n$-braid $\beta$ which closes to the unknot is conjugate to $\sigma_{1} \sigma_{2} \ldots \sigma_{n-1}$.

TheOREM 3. Any positive permutation $n$-braid $\beta$ which closes to the trefoil is conjugate to $\sigma_{1}^{3} \sigma_{2} \ldots \sigma_{n-1}$.

Proof of Theorem 2. Each generator $\sigma_{i}$ must appear at least once in $\beta$, otherwise its closure is disconnected. Since its closure has genus 0 the braid $\beta$ has $n-1$ crossings, and so each generator appears exactly once.

It is enough to manipulate the braid cyclically, as such manipulations can be realised as conjugacies. We can represent $\beta$ up to conjugacy by writing the generators $\sigma_{1}, \ldots, \sigma_{n-1}$ in the appropriate order around a circle. Each generator appears exactly once. To prove the theorem we use the braid commutation relations to rearrange the generators in ascending order round the circle.

Assume by induction on $j$ that the generators $\sigma_{1}, \ldots, \sigma_{j}$ occur consecutively in order. Then any generator $\sigma_{k}$ lying on the circle between $\sigma_{j}$ and $\sigma_{j+1}$ has $k>j+1$. These generators then commute with each of $\sigma_{1}, \ldots, \sigma_{j}$, and can be moved past them to leave $\sigma_{j+1}$ immediately after $\sigma_{j}$. The process finishes when all generators are in consecutive order. 
Proof of Theorem 3. Again represent generators on a circle. Each generator $\sigma_{1}, \ldots, \sigma_{n-1}$ must occur at least once, otherwise the closed braid splits. Since the trefoil has genus 1 the braid has $n+1$ crossings. So either two generators $\sigma_{i}$ and $\sigma_{j}$ each occur twice, or one, $\sigma_{i}$ say, occurs three times, and the other generators occur once only.

Either $\sigma_{i-1}$ or $\sigma_{i+1}$ must occur between two occurrences of $\sigma_{i}$ in $\beta$, otherwise it can be rewritten with two consecutive occurrences of $\sigma_{i}$. This is not possible for a permutation braid, since pairs of strings cross at most once. If $\sigma_{i}$ occurs three times then $\sigma_{i-1}$ lies between one pair of occurrences of $\sigma_{i}$ and $\sigma_{i+1}$ between the other pair. We can then move all generators except $\sigma_{i+1}$ past these last two occurrences of $\sigma_{i}$ to write $\beta$ with a consecutive sequence $\sigma_{i} \sigma_{i+1} \sigma_{i}$. Change this to $\sigma_{i+1} \sigma_{i} \sigma_{i+1}$ by the braid relation to write $\beta$ with $\sigma_{i}$ and $\sigma_{i+1}$ each appearing twice.

We may thus assume that two generators $\sigma_{i}$ and $\sigma_{j}$ each occur twice in $\beta$, with $j>i$. If $j>i+1$ then $\sigma_{i+1}$ occurs only once. We can then collect all generators $\sigma_{k}$ with $k>i+1$ at the two ends of the braid word, and combine them at the end of the word by cycling so as to write a conjugate braid in the form $A B$ where $B$ is a product of generators $\sigma_{k}$ with $k>i+1$, and includes $\sigma_{j}$ twice, while $A$ is a product with $k \leq i+1$, and includes $\sigma_{i}$ twice. The closure of the braid then has three components and not one.

Hence $\beta$ must contain $\sigma_{i}$ and $\sigma_{i+1}$ twice each. Furthermore their occurrences must be interleaved, otherwise we can cycle the braid and commute elements to separate it as a product of generators $\sigma_{k}$ with $k \leq i$ and those with $k>i$, and its closure will again have three components.

We shall prove, by induction on $i$, that any positive braid with two interleaved occurrences of $\sigma_{i}$ and $\sigma_{i+1}$, and single occurrences of all other generators, is conjugate to $\sigma_{1}^{3} \sigma_{2} \ldots \sigma_{n-1}$.

We can assume, by cycling, that the single occurrence of $\sigma_{i-1}$ does not lie between the two occurrences of $\sigma_{i}$. We can move all further generators except $\sigma_{i+1}$ past $\sigma_{i}$ so as to write $\sigma_{i} \sigma_{i+1} \sigma_{i}$ consecutively. The remaining occurrence of $\sigma_{i+1}$ can be moved round the circle past any other generator except the single $\sigma_{i+2}$. It can then be moved one way or other round the circle to reach this block of three generators, giving either $\sigma_{i} \sigma_{i+1} \sigma_{i} \sigma_{i+1}$ or $\sigma_{i+1} \sigma_{i} \sigma_{i+1} \sigma_{i}$. The braid relation then gives a consecutive block of either $\sigma_{i} \sigma_{i} \sigma_{i+1} \sigma_{i}$ or $\sigma_{i} \sigma_{i+1} \sigma_{i} \sigma_{i}$. The single $\sigma_{i-1}$ now lies between two occurrences of $\sigma_{i}$ on the circle. Any intervening generators commute with $\sigma_{i}$ and can be moved out to leave $\sigma_{i} \sigma_{i-1} \sigma_{i}$, which can be converted to $\sigma_{i-1} \sigma_{i} \sigma_{i-1}$. The cyclic braid now has two interleaving occurrences of $\sigma_{i-1}$ and $\sigma_{i}$.

The result follows by induction on $i$, once we establish it for $i=1$. In this case the argument above provides a block of either $\sigma_{1} \sigma_{1} \sigma_{2} \sigma_{1}$ or $\sigma_{1} \sigma_{2} \sigma_{1} \sigma_{1}$ 
on the circle. Since $\sigma_{1}$ commutes with all generators except $\sigma_{2}$ we can move the right-hand occurrences of $\sigma_{1}$ round the circle to give a block $\sigma_{1} \sigma_{1} \sigma_{1} \sigma_{2}$. The remaining generators can then be put in ascending order as in the proof of Theorem 2.

A quick check on the possible values of the writhe for the $(n-1)$ ! positive permutation braids with $n \leq 4$ which close to a knot shows that in this range conjugacy is determined simply by writhe, in view of Theorems 2 and 3 . Positive permutation braids with $n+3$ crossings arise first when $n=5$. A direct check on the corresponding braids shows that in this case too the writhe is sufficient.

TheORem 4. Positive permutation braids on $n$ strings which close to a knot are conjugate if and only if they have the same number of crossings, when $n \leq 5$.

Tables of these braids for $n=3,4,5$, and the corresponding permutations, are included below.

When $n=3$ there are just two braids which both close to the unknot.

\begin{tabular}{ccc}
\hline Permutation & Braid word & $\begin{array}{c}\text { Number of } \\
\text { crossings }\end{array}$ \\
\hline$(123)$ & $\sigma_{2} \sigma_{1}$ & 2 \\
$(132)$ & $\sigma_{1} \sigma_{2}$ & 2 \\
\hline
\end{tabular}

When $n=4$ there are two conjugacy classes. The braids with writhe 3 close to the unknot, and those with writhe 5 to the trefoil.

\begin{tabular}{ccc}
\hline Permutation & Braid word & $\begin{array}{c}\text { Number of } \\
\text { crossings }\end{array}$ \\
\hline$(1234)$ & $\sigma_{3} \sigma_{2} \sigma_{1}$ & 3 \\
$(1243)$ & $\sigma_{2} \sigma_{1} \sigma_{3}$ & 3 \\
$(1342)$ & $\sigma_{1} \sigma_{3} \sigma_{2}$ & 3 \\
$(1432)$ & $\sigma_{1} \sigma_{2} \sigma_{3}$ & 3 \\
$(1324)$ & $\sigma_{2} \sigma_{1} \sigma_{3} \sigma_{2} \sigma_{1}$ & 5 \\
$(1423)$ & $\sigma_{1} \sigma_{2} \sigma_{1} \sigma_{3} \sigma_{2}$ & 5 \\
\hline
\end{tabular}

When $n=5$ there are three conjugacy classes. The braids with writhe $4=n-1$ close to the unknot and those with writhe 6 close to the trefoil. Those with writhe $8=n+3$ all close to the $(2,5)$ torus knot. 


\begin{tabular}{ccc}
\hline Permutation & Braid word & $\begin{array}{c}\text { Number of } \\
\text { crossings }\end{array}$ \\
\hline$(12345)$ & $\sigma_{4} \sigma_{3} \sigma_{2} \sigma_{1}$ & 4 \\
$(12354)$ & $\sigma_{3} \sigma_{2} \sigma_{1} \sigma_{4}$ & 4 \\
$(12453)$ & $\sigma_{2} \sigma_{1} \sigma_{4} \sigma_{3}$ & 4 \\
$(12543)$ & $\sigma_{2} \sigma_{1} \sigma_{3} \sigma_{4}$ & 4 \\
$(13452)$ & $\sigma_{1} \sigma_{4} \sigma_{3} \sigma_{2}$ & 4 \\
$(13542)$ & $\sigma_{1} \sigma_{3} \sigma_{2} \sigma_{4}$ & 4 \\
$(14532)$ & $\sigma_{1} \sigma_{2} \sigma_{4} \sigma_{3}$ & 4 \\
$(15432)$ & $\sigma_{1} \sigma_{2} \sigma_{3} \sigma_{4}$ & 4 \\
$(12435)$ & $\sigma_{3} \sigma_{2} \sigma_{4} \sigma_{3} \sigma_{2} \sigma_{1}$ & 6 \\
$(12534)$ & $\sigma_{2} \sigma_{3} \sigma_{2} \sigma_{1} \sigma_{4} \sigma_{3}$ & 6 \\
$(13245)$ & $\sigma_{2} \sigma_{1} \sigma_{4} \sigma_{3} \sigma_{2} \sigma_{1}$ & 6 \\
$(13254)$ & $\sigma_{2} \sigma_{1} \sigma_{3} \sigma_{2} \sigma_{1} \sigma_{4}$ & 6 \\
$(13524)$ & $\sigma_{3} \sigma_{2} \sigma_{1} \sigma_{4} \sigma_{3} \sigma_{2}$ & 6 \\
$(14253)$ & $\sigma_{2} \sigma_{1} \sigma_{3} \sigma_{2} \sigma_{4} \sigma_{3}$ & 6 \\
$(14352)$ & $\sigma_{1} \sigma_{3} \sigma_{2} \sigma_{4} \sigma_{3} \sigma_{2}$ & 6 \\
$(14523)$ & $\sigma_{1} \sigma_{2} \sigma_{1} \sigma_{4} \sigma_{3} \sigma_{2}$ & 6 \\
$(15342)$ & $\sigma_{1} \sigma_{2} \sigma_{3} \sigma_{2} \sigma_{4} \sigma_{3}$ & 6 \\
$(15423)$ & $\sigma_{1} \sigma_{2} \sigma_{1} \sigma_{3} \sigma_{2} \sigma_{4}$ & 6 \\
$(13425)$ & $\sigma_{2} \sigma_{3} \sigma_{2} \sigma_{1} \sigma_{4} \sigma_{3} \sigma_{2} \sigma_{1}$ & 8 \\
$(14235)$ & $\sigma_{1} \sigma_{3} \sigma_{2} \sigma_{1} \sigma_{4} \sigma_{3} \sigma_{2} \sigma_{1}$ & 8 \\
$(14325)$ & $\sigma_{2} \sigma_{1} \sigma_{3} \sigma_{2} \sigma_{4} \sigma_{3} \sigma_{2} \sigma_{1}$ & 8 \\
$(15234)$ & $\sigma_{1} \sigma_{2} \sigma_{3} \sigma_{2} \sigma_{1} \sigma_{4} \sigma_{3} \sigma_{2}$ & 8 \\
$(15243)$ & $\sigma_{1} \sigma_{2} \sigma_{1} \sigma_{3} \sigma_{2} \sigma_{4} \sigma_{3} \sigma_{2}$ & 8 \\
\hline & $\sigma_{1} \sigma_{2} \sigma_{1} \sigma_{3} \sigma_{2} \sigma_{1} \sigma_{4} \sigma_{3}$ & 8 \\
\hline & & \\
$(15324)$ & & 6 \\
\hline
\end{tabular}

Having looked among the closures of positive permutation braids at knots with the smallest number of crossings, in Theorems 2 and 3 , we now turn briefly to those with the largest possible number.

The largest number of crossings in any positive permutation braid in $B_{n}$ is $\frac{1}{2} n(n-1)$, which occurs for the fundamental half-twist braid $\Delta_{n}$. If the closure is to be a knot the largest number of crossings is $\frac{1}{2} n(n-1)-\left[\frac{1}{2}(n-1)\right]$.

THEOREM 5. Every positive permutation braid with $\frac{1}{2} n(n-1)-\left[\frac{1}{2}(n-1)\right]$ crossings which closes to a knot is conjugate to $\Delta_{n} \sigma_{1}^{-1} \sigma_{2}^{-1} \ldots \sigma_{k}^{-1}$ where $k=\left[\frac{1}{2}(n-1)\right]$.

Proof. Take $n=2 k+1$ or $n=2 k+2$, so that $k=\left[\frac{1}{2}(n-1)\right]$, and let $\beta$ be a positive permutation braid with $\frac{1}{2} n(n-1)-k$ crossings which closes to a knot. Then $\beta$ has a complementary positive permutation braid 
$\gamma$ in $\Delta_{n}$, with $\beta \gamma=\Delta_{n}$. The braid $\gamma$ has $k$ crossings. Since $\beta=\Delta_{n} \gamma^{-1}$ closes to a knot the $k$ crossings in $\gamma^{-1}$ must be used to connect up the $k+1$ components in $\widehat{\Delta}_{n}$. Hence the $k$ generators in $\gamma^{-1}$ must all be different. When $n=2 k+2$ the generator $\sigma_{k+1}$ cannot occur, since this connects two strings which are already in the same component of $\widehat{\Delta}_{n}$, and more generally $\sigma_{j}$ and $\sigma_{n-j}$ cannot both occur, for any $j$, as they both connect the same two components. In particular the generators $\sigma_{k}$ and $\sigma_{k+1}$ cannot both occur when $n=2 k+1$.

The generators in $\gamma$ then belong to two mutually commuting sets, those from $\sigma_{1}$ up to $\sigma_{k}$ and those from $\sigma_{k+1}$ up to $\sigma_{n-1}$. Write $\beta$ on a circle, with one block of generators together as $\Delta_{n}$ and then the $k$ generators of $\gamma^{-1}$. Move all generators $\sigma_{j}$ with $j>k$ to the extreme right in $\gamma^{-1}$ and then round the circle to the left of $\Delta_{n}$. Now move them past $\Delta_{n}$, when each $\sigma_{j}$ is converted to $\sigma_{n-j}$. Since $\sigma_{j}$ and $\sigma_{n-j}$ did not both occur in $\gamma$ we get a braid $\Delta_{n} \alpha^{-1}$ conjugate to $\beta$ in which $\sigma_{1}, \ldots, \sigma_{k}$ each occurs exactly once in $\alpha$.

Following the method of Theorem 2 we can arrange the $k$ generators in $\alpha$ in any order up to conjugacy, once we know how to move any generator $\sigma_{j}$ from the left to the right of $\alpha^{-1}$ by conjugacy. This can be done by taking it twice round the circle as follows. First move $\sigma_{j}$ to the left of $\Delta_{n}$, when it becomes $\sigma_{n-j}$. Then move it round the circle to the end of the word. It can then be moved left past all the remaining generators of $\alpha^{-1}$, and past $\Delta_{n}$ once more, to become $\sigma_{j}$. Finally, move this round the circle to the right-hand end of $\alpha^{-1}$.

Consequently, $\beta$ is conjugate to $\Delta_{n} \sigma_{1}^{-1} \sigma_{2}^{-1} \ldots \sigma_{k}^{-1}$.

3. Non-conjugacy results. When $n=6$ it is possible to have two positive permutation braids with the same number of crossings which close to different knots. The permutations (124536), with braid $\sigma_{3} \sigma_{4} \sigma_{3} \sigma_{2} \sigma_{5} \sigma_{4} \sigma_{3} \sigma_{2} \sigma_{1}$, and (132546), with braid $\sigma_{2} \sigma_{1} \sigma_{4} \sigma_{3} \sigma_{5} \sigma_{4} \sigma_{3} \sigma_{2} \sigma_{1}$, close to the $(2,5)$ torus knot and the sum of two trefoils respectively, so writhe no longer determines conjugacy.

In [9], Hadji gave examples of two non-conjugate positive permutation braids with $n=16$, each closing to the same connected sum of three knots.

In fact, non-conjugate positive permutation braids which close to the same knot show up first when $n=6$.

THEOREM 6. The positive permutation braids in $S_{6}^{+}$with permutations (165324) and (152643) have the same closure but are not conjugate.

Proof. The braids, shown below, can be written $\beta=\sigma_{1} \sigma_{3} \sigma_{5} \sigma_{2} \sigma_{4} \sigma_{1} \sigma_{3} \sigma_{2} \sigma_{1}$ and $\gamma=\sigma_{2} \sigma_{4} \sigma_{3} \sigma_{5} \sigma_{2} \sigma_{4} \sigma_{1} \sigma_{3} \sigma_{2}$ respectively. Both of these can be reduced by Markov moves to the 4 -braid $\sigma_{1} \sigma_{3} \sigma_{2} \sigma_{1} \sigma_{3} \sigma_{2} \sigma_{1}$, so both close to the $(2,5)$ torus knot. 

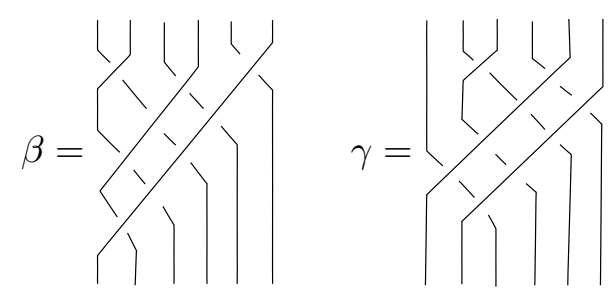

The squares of the two braids are shown here with the strings which form one component of the closure emphasised.
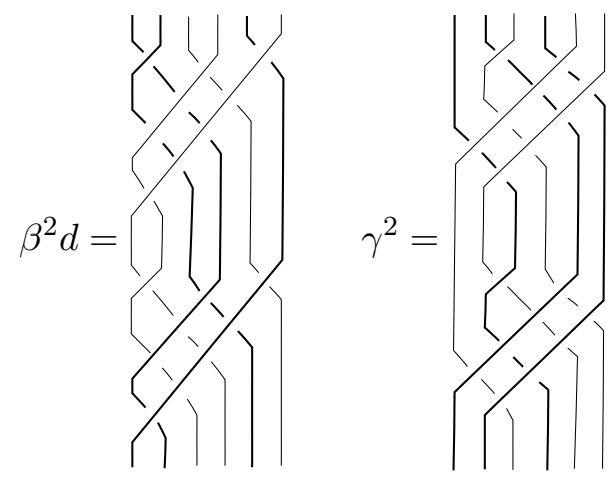

If $\beta$ and $\gamma$ are conjugate then so are $\beta^{2}$ and $\gamma^{2}$. Now the closure of $\beta^{2}$ is a link with two components, each of which turns out to be the trefoil knot, while the two components of the closure of $\gamma^{2}$ are trivial knots. Hence $\beta^{2}$ and $\gamma^{2}$ are not conjugate.

An alternative check can be made by calculating the 2 -variable Alexander polynomial of the link consisting of the closure of $\beta$ and its axis. If $\beta$ is conjugate to $\gamma$ this link is isotopic to the closure of $\gamma$ and its axis. Its polynomial is in general the characteristic polynomial of the reduced Burau matrix of the braid [11]. For $\beta$ above, the polynomial is

$$
t^{9} x^{5}+t^{7} x^{4}+t^{5} x^{3}+t^{4} x^{2}+t^{2} x+1
$$

which differs, up to multiples of $\pm t^{i} x^{j}$, from the polynomial

$$
t^{9} x^{5}+t^{7} x^{4}+\left(2 t^{5}-t^{4}\right) x^{3}+\left(2 t^{4}-x^{5}\right) x^{2}+t^{2} x+1
$$

for $\gamma$.

Other tests for conjugacy, which also rely in effect on invariants of a closed braid in a solid torus, can be used to give a contrasting result to Theorem 3 about positive braids which close to the trefoil, when we do not restrict to positive permutation braids.

THEOREM 7. If $\beta \in B_{n}^{+}$closes to the trefoil knot then $\beta$ is conjugate to $\beta(i)=\sigma_{1} \sigma_{2} \ldots \sigma_{i-1} \sigma_{i}^{3} \sigma_{i+1} \ldots \sigma_{n-1}$ for some $i$. Two such braids $\beta(i), \beta(k)$ are conjugate if and only if $k=i$ or $k=n-i$. 
REMARK. When $n=4$ the braids are examples of the construction of Murasugi and Thomas, [13]. They show that the braids $\sigma_{1}^{p} \sigma_{2}^{q} \sigma_{3}^{r}$ and $\sigma_{1}^{p} \sigma_{2}^{r} \sigma_{3}^{q}$, with $p, q, r$ odd, which close to isotopic knots, are not conjugate when $q \neq r$. Their proof uses the exceptional homomorphism from $B_{4}$ to $B_{3}$ defined by $\sigma_{1}, \sigma_{3} \mapsto \sigma_{1}, \sigma_{2} \mapsto \sigma_{2}$, observing that the braids map to $\sigma_{1}^{p+r} \sigma_{2}^{q}$ and $\sigma_{1}^{p+q} \sigma_{2}^{r}$, which close to links with different linking numbers.

\section{Proof of Theorem $\%$.}

1. Conjugacy. The only difference from the argument of Theorem 3 is that one generator $\sigma_{i}$ may occur three times, with $\sigma_{i-1}$ and $\sigma_{i+1}$ both lying on the circle between the same pair of occurrences of $\sigma_{i}$. Then all three occurrences of $\sigma_{i}$ can be moved together and remain as a block on the circle, while the other generators are put in consecutive order, as in Theorem 2 . This shows that every such braid is conjugate to some $\beta(i)$. To see that the braids $\beta(i)$ and $\beta(n-i)$ are conjugate, first conjugate $\beta(i)$ by $\Delta_{n}$, taking $\sigma_{i}^{3}$ to $\sigma_{n-i}^{3}$, and then rearrange as above.

2. Non-conjugacy. Any closed braid represents an element in the framed Homfly skein of closed braids in the annulus [12]. The closure of $\sigma_{1} \sigma_{2} \ldots \sigma_{k-1}$ represents an element $A_{k}$. The skein itself admits a commutative product, represented by the closures of split braids. The subspace spanned by the closure of braids in $B_{n}$ has a basis consisting of monomials $A_{i_{1}} \ldots A_{i_{k}}$ with $i_{1}+\cdots+i_{k}=n$. Coefficients in the skein can be taken as integer polynomials in a variable $z$. In the Homfly skein of braids before closure, we have $\sigma_{i}^{3}=$ $c(z) \sigma_{i}+d(z)$, for some fixed non-zero polynomials $c(z), d(z)$, so that $\beta(i)=$ $c(z) \sigma_{1} \ldots \sigma_{n-1}+d(z) \sigma_{1} \ldots \sigma_{i-1} \sigma_{i+1} \ldots \sigma_{n-1}$ in this skein. Its closure then represents $c(z) A_{n}+d(z) A_{i} A_{n-i}$ in the skein of the annulus.

If $\beta(i)$ and $\beta(k)$ are conjugate then they have the same closure in the annulus. Then

$$
c(z) A_{n}+d(z) A_{i} A_{n-i}=c(z) A_{n}+d(z) A_{k} A_{n-k},
$$

and hence $A_{i} A_{n-i}=A_{k} A_{n-k}$. The monomials form a basis in the skein of the annulus, so $k=i$ or $k=n-i$.

REMARK. This same calculation can be used to show that the Conway polynomial of the closure of $\beta(i)$ and its axis differs from that of $\beta(k)$ and its axis except when $k=i$ or $n-i$.

4. Conjugacy classes for 6 and more strings. We have a short Maple procedure to list the positive permutation braids on $n$ strings which close to knots, according to their number of crossings.

The case $n=6$. When $n=6$ this list contains 16 positive permutation braids with 5 crossings, 32 with 7 crossings, 44 with 9 crossings, 22 with 11 crossings and 6 with 13 crossings. 
By Theorems 2, 3 and 5 those with 5,7 or 13 crossings form complete conjugacy classes, and represent the trivial knot, the trefoil and the $(3,5)$ torus knot respectively. An inductive count shows that there are in general $2^{n-2}$ braids in $S_{n}^{+}$which represent the trivial knot.

Among the braids with 9 crossings there is one conjugacy class consisting of 4 braids which close to the sum of two trefoils, and two classes of braids which close to the $(2,5)$ torus knot. There are just 2 braids, $\gamma=\sigma_{2} \sigma_{4} \sigma_{3} \sigma_{5} \sigma_{2} \sigma_{4} \sigma_{1} \sigma_{3} \sigma_{2}$ and its conjugate by the half-twist, in the conjugacy class of the braid $\gamma$ discussed in Theorem 6 , while the remaining 38 braids are conjugate to $\beta=\sigma_{1} \sigma_{3} \sigma_{5} \sigma_{2} \sigma_{4} \sigma_{1} \sigma_{3} \sigma_{2} \sigma_{1}$.

The braids with 11 crossings fall into two conjugacy classes, one containing 6 braids which close to the $(3,4)$ torus knot, and the other containing 16 braids which close to the $(2,7)$ torus knot.

The case $n=7$. When $n=7$ there are 32 positive permutation braids with 6 crossings, 88 with 8 crossings, 176 with 10 crossings, 202 with 12 crossings, 134 with 14 crossings, 70 with 16 crossings and 18 with 18 crossings.

Again those with 6,8 or 18 crossings represent complete conjugacy classes; we have not attempted to analyse the other classes any further, or to consider in detail any cases where $n>7$.

\section{References}

[1] E. Artin, Theory of braids, Ann. of Math. 48 (1947), 101-126.

[2] J. S. Birman, Braids, Links, and Mapping Class Groups, Ann. of Math. Stud. 82, Princeton Univ. Press, Princeton, NJ, 1974.

[3] E. S. Elrifai, Positive braids and Lorenz links, PhD dissertation, Univ. of Liverpool, 1988.

[4] E. S. Elrifai and M. Benkhalifa, On the conjugacy problem of positive braids, preprint, King Khalid Univ., 2003.

[5] E. S. Elrifai and H. R. Morton, Algorithms for positive braids, Quart. J. Math. Oxford Ser. (2) 45 (1994), 479-497.

[6] T. Fiedler, Gauss Diagram Invariants for Knots and Links, Math. Appl. 532, Kluwer, Dordrecht, 2001.

[7] N. Franco and J. González-Meneses, Conjugacy problem for braid groups and Garside groups, J. Algebra 266 (2003), 112-132.

[8] F. A. Garside, The braid group and other groups, Quart. J. Math. Oxford Ser. (2) 20 (1969), 235-254.

[9] R. J. Hadji, The conjugacy problem for positive permutation braids inducing an n-cycle, MSc mini-dissertation, Univ. of Liverpool, 1999.

[10] T. Hall, private communication, 2002.

[11] H. R. Morton, Infinitely many fibred knots with the same Alexander polynomial, Topology 17 (1978), 101-104. 
[12] H. R. Morton, Power sums and Homfly skein theory, in: Invariants of Knots and 3-manifolds (Kyoto, 2001), Geom. Topol. Monogr. 4, Geom. Topol. Publ., Coventry, 2002, 235-244.

[13] K. Murasugi and R. S. D. Thomas, Isotopic closed nonconjugate braids, Proc. Amer. Math. Soc. 33 (1972), 137-139.

Department of Mathematical Sciences

University of Liverpool

Peach St., Liverpool, L69 7ZL, England

E-mail: morton@liv.ac.uk

rjhadji@o2.co.uk

Received 10 June 2004;

in revised form 25 April 2005 